\title{
Measurements of the Total Cross Section for the Scattering of Polarized Neutrons from Polarized ${ }^{3} \mathrm{He}$
}

\author{
C. D. Keith, ${ }^{*}$ C. R. Gould, D. G. Haase and M. L. Seely \\ North Carolina State University, Raleigh, NC 27695, USA and Triangle Universities Nuclear \\ Laboratory, Durham, NC 27ro8, USA \\ P. R. Huffman, ${ }^{\dagger}$ N. R. Roberson, W. Tornow and W. S. Wilburn \\ Duke University, Durham, NC 27708, USA and Triangle Universities Nuclear Laboratory, \\ Durham, NC 27708, USA
}

\begin{abstract}
Measurements of polarized neutron-polarized ${ }^{3} \mathrm{He}$ scattering are reported. The target consisted of cryogenically-polarized solid ${ }^{3} \mathrm{He}$, thickness 0.04 atom/b and polarization $\sim 0.4$. Polarized neutrons were produced via the ${ }^{3} \mathrm{H}(\vec{p}, \vec{n})^{3} \mathrm{He}$ or ${ }^{2} \mathrm{H}(\vec{d}, \vec{n})^{3} \mathrm{He}$ polarization-transfer reactions. The longitudinal and transverse total cross-section differences $\Delta \sigma_{L}$ and $\Delta \sigma_{T}$ were measured for incident neutron energies $2-8 \mathrm{MeV}$. The results are compared to phase-shift predictions based on four different analyses of $n-{ }^{3} \mathrm{He}$ scattering. The best agreement is obtained with a recent $R$-matrix analysis of $A=4$ scattering and reaction data, lending strong support to the ${ }^{4} \mathrm{He}$ level scheme obtained in that analysis. Discrepancies with other phase-shift parameterizations of $n-{ }^{3} \mathrm{He}$ scattering exist, attributable in most instances to one or two particular partial waves.
\end{abstract}

\footnotetext{
*Present address: Indiana University Cyclotron Facility, Bloomington, IN 47408

${ }^{\dagger}$ Present address: Physics Department, Harvard University, Cambridge, MA 02138
} 


\section{INTRODUCTION}

Recent computational advances in the field of few-nucleon dynamics have fueled renewed interest in the three-nucleon and four-nucleon systems [1]. Exact bound-state calculations utilizing realistic, meson-exchange forces are now possible for both the $3 \mathrm{~N}$ and $4 \mathrm{~N}$ systems. Similar calculations are currently available for the $3 \mathrm{~N}$ continuum, and extension to the $4 \mathrm{~N}$ continuum is under active investigation.

The continuum calculations may prove especially revealing, because here exists a fundamental difference between the three- and four-nucleon systems: three-nucleon systems have no excited states whereas the four-nucleon system has many. These states (resonances) may exhibit sensitivity to the dynamics of the nucleon-nucleon interaction and their modification in the presence of the nuclear medium. However, confirming the existence, and determining the quantum numbers of these resonances is a challenging experimental problem. The level scheme proposed as part of a recent review article [2] has 15 levels at excitations 20-30 MeV above the ground state. For the most part these resonances do not appear as sharp structure in any scattering or reaction observable, and polarization measurements are essential for determining the scattering amplitudes.

While there have been many studies of single polarization observables in $4 \mathrm{~N}$ systems, there is very little data with both polarized target and polarized beam. Only two measurements have been reported. In 1966, Passell and Schermer measured the transmission of polarized thermal neutrons through a polarized ${ }^{3} \mathrm{He}$ target [3], and more recently, Alley and Knutsen studied $p{ }^{3} \mathrm{He}$ spin-correlation data [4. In the first experiment, the thermal cross section is completely dominated by a single $\left(0^{+}\right)$subthreshold resonance in the ${ }^{4} \mathrm{He}$ compound nucleus, and no information on the higher-energy resonances was obtained. The second experiment covered a much broader region in the $A=4$ continuum, but was sensitive only to the isotriplet scattering states. No previous polarized target-polarized beam experiment has fully explored a wide range of $4 \mathrm{~N}$ excited states.

In this paper we report measurements of the longitudinal and transverse neutron total cross-section differences $\Delta \sigma_{L}$ and $\Delta \sigma_{T}$. These two spin observables are directly related to the forward elastic-scattering amplitude through the optical theorem [5]. As such, they allow for a simple interpretation in terms of the properties of the scattering states. The measurements were performed at energies corresponding to excitations $22-27 \mathrm{MeV}$ above the ${ }^{4} \mathrm{He}$ ground state, where a number of broad, negative-parity levels are believed to exist.

The remainder of this paper is organized as follows. In Sec. II we discuss the basic principles behind the measurements. The polarized target and polarized beam are described in Sections IIIA and IIIB, respectively. The experimental procedure and method of data analysis are described in Sec. IV. The $\Delta \sigma_{L}$ and $\Delta \sigma_{T}$ results are presented in Sec. $\nabla$ and compared to four separate sets of $n-{ }^{3} \mathrm{He}$ phase shifts in Sec. VI. Our conclusions are summarized in Sec. VII. A preliminary version of the $\Delta \sigma_{T}$ results has appeared elsewhere [6].

\section{THEORY}

The formalism necessary to describe the neutron total cross section for polarized target and polarized beam has been developed and discussed in an earlier paper [5]. For the sake of clarity we briefly review the results of that paper in the following section. 
The parity-conserving, time-reversal invariant part of the forward elastic scattering amplitude allows the neutron total cross section for spin- $1 / 2$ nuclei to be expressed as

$$
\sigma_{\text {tot }}=\sigma_{0}+\frac{1}{2} \Delta \sigma_{L} P_{n}^{z} P_{t}^{z}+\frac{1}{2} \Delta \sigma_{T}\left(P_{n}^{x} P_{t}^{x}+P_{n}^{y} P_{t}^{y}\right)
$$

Here $\sigma_{0}$ is the unpolarized neutron total cross section, $P_{n}^{i}\left(P_{t}^{i}\right)$ is the $i$ th projection of the beam (target) polarization axis, and $\Delta \sigma_{L}$ and $\Delta \sigma_{T}$ are the longitudinal and transverse total cross-section differences. The latter may be represented pictorially as

$$
\Delta \sigma_{L}=\sigma_{t o t}(\vec{\rightarrow})-\sigma_{t o t}(\vec{\leftarrow})
$$

and

$$
\Delta \sigma_{T}=\sigma_{\text {tot }}(\uparrow \uparrow)-\sigma_{\text {tot }}(\uparrow \downarrow) .
$$

The measurements of $\Delta \sigma_{L}$ and $\Delta \sigma_{T}$ reported here were performed separately, with both beam and target polarized along axes longitudinal to the incident beam direction (i.e. along the $z$ axis) or transverse to the beam direction (i.e. along the $y$ axis). Hence in subsequent discussion, the superscripts used to indicate the polarization axes will be dropped.

An accurate determination of the $\Delta \sigma_{L}$ and $\Delta \sigma_{T}$ observables does not require an absolute measurement of the total cross section. Rather, the cross-section differences may be extracted from transmission measurements of polarized neutrons through the polarized target. The attenuation of an incident beam of $N_{0}$ polarized neutrons due to a polarized target of areal density $\tau$ will be

$$
N_{ \pm} / N_{0}=\exp \left[-\tau\left(\sigma_{0} \pm \frac{1}{2} \Delta \sigma_{L} P_{n} P_{t}\right)\right]
$$

when both the beam and target are longitudinally polarized, or

$$
N_{ \pm} / N_{0}=\exp \left[-\tau\left(\sigma_{0} \pm \frac{1}{2} \Delta \sigma_{T} P_{n} P_{t}\right)\right]
$$

when beam and target are polarized in the transverse direction. The \pm signs are used to indicate whether the beam and target spins are polarized parallel $(+)$ or antiparallel $(-)$ to one another. By periodically reversing the spin of the beam (or target), one may observe an asymmetry in the attenuation of the beam. This neutron-transmission or spin-spin asymmetry is defined as

$$
\begin{aligned}
\varepsilon_{L, T} & =\frac{N_{+}-N_{-}}{N_{+}+N_{-}} \\
& =\tanh \left[-\frac{1}{2} \Delta \sigma_{L, T} P_{n} P_{t} \tau\right] \\
& \approx-\frac{1}{2} \Delta \sigma_{L, T} P_{n} P_{t} \tau .
\end{aligned}
$$

\footnotetext{
${ }^{1}$ Note that our definition of parallel minus antiparallel is exactly opposite to the convention commonly used for nucleon-nucleon scattering.
} 
Because the observed values of $\varepsilon$ are typically of order $10^{-3}$, the error introduced by replacing the hyperbolic tangent with its argument in Eq. (6) is negligible. In writing Eq. (6) we have assumed that the incident neutron flux $N_{0}$ is unaffected by reversing the spin.

Because they are directly related to the forward scattering amplitude via the optical theorem, $\sigma_{0}, \Delta \sigma_{L}$, and $\Delta \sigma_{T}$ are not sensitive to interference effects between the various partial waves. In principle this allows for a much simpler interpretation of the scattering and reaction processes. The $n-{ }^{3} \mathrm{He}$ total cross sections can be expressed as linear sums of "partial-wave" cross sections $\sigma\left(J, l, s, l^{\prime}, s^{\prime}\right)$, where

$$
\sigma\left(J, l, s, l^{\prime}, s^{\prime}\right)=\operatorname{Re}\left\{\frac{\pi}{2 k^{2}}(2 J+1)\left[\delta_{l l^{\prime}} \delta_{s s^{\prime}}-S_{l l^{\prime} s s^{\prime}}^{J}\right]\right\}
$$

Here $J, l, s\left(J, l^{\prime}, s^{\prime}\right)$ are the incoming (outgoing) total, orbital, and channel-spin angular momenta, $k$ is the neutron center-of-mass wave number, and $S_{l l^{\prime} s s^{\prime}}^{J}$ is the elastic scattering matrix element that describes transitions from the initial neutron channel $(J, l, s)$ to the final neutron channel $\left(J, l^{\prime}, s^{\prime}\right)$. For partial waves up to $l=1$, we find

$$
\begin{aligned}
\sigma_{0}=\sigma & \left({ }^{1} S_{0}\right)+\sigma\left({ }^{3} S_{1}\right)+\sigma\left({ }^{1} P_{1}\right) \\
& +\sigma\left({ }^{3} P_{0}\right)+\sigma\left({ }^{3} P_{1}\right)+\sigma\left({ }^{3} P_{2}\right) \\
\Delta \sigma_{L}= & -2 \sigma\left({ }^{1} S_{0}\right)+\frac{2}{3} \sigma\left({ }^{3} S_{1}\right)-2 \sigma\left({ }^{1} P_{1}\right) \\
& -2 \sigma\left({ }^{3} P_{0}\right)+2 \sigma\left({ }^{3} P_{1}\right)+\frac{2}{5} \sigma\left({ }^{3} P_{2}\right) \\
& +\frac{4}{3} \sqrt{2} \sigma\left({ }^{3} S_{1}-{ }^{3} D_{1}\right),
\end{aligned}
$$

and

$$
\begin{aligned}
\Delta \sigma_{T}= & -2 \sigma\left({ }^{1} S_{0}\right)+\frac{2}{3} \sigma\left({ }^{3} S_{1}\right)-2 \sigma\left({ }^{1} P_{1}\right) \\
& +2 \sigma\left({ }^{3} P_{0}\right)+\frac{4}{5} \sigma\left({ }^{3} P_{2}\right)-\frac{2}{3} \sqrt{2} \sigma\left({ }^{3} S_{1}-{ }^{3} D_{1}\right) .
\end{aligned}
$$

A negative coefficient simply implies that the cross section for this particular wave is greater when the beam and target spins are antiparallel to one another.

Detailed predictions of the total cross-section differences $\Delta \sigma_{L}$ and $\Delta \sigma_{T}$ for $\vec{n}{ }^{3} \overrightarrow{\mathrm{He}}$ scattering were presented in an earlier paper [5]. These calculations were based on three separate analyses of $n-{ }^{3} \mathrm{He}$ scattering and reaction data [7,9], as well as a microscopic resonatinggroup model calculation of the $4 \mathrm{~N}$ excited states [10]. It was observed that, although the different sets of phase shifts provide adequate descriptions of pre-existing data, they predict quantitatively different values of both $\Delta \sigma_{L}$ and $\Delta \sigma_{T}$ in the present region of interest. In most instances these discrepancies could be attributed to one or two partial waves. Based on this observation, we concluded that comprehensive measurements of both $\Delta \sigma_{L}$ and $\Delta \sigma_{T}$, in combination with the unpolarized neutron total cross section $\sigma_{0}$, could be used to extract specific information about the partial-wave content of the resonating ${ }^{4} \mathrm{He}$ compound nucleus. 


\section{EXPERIMENTAL APPARATUS}

\section{A. Polarized ${ }^{3}$ He Target}

A brief description of the polarized solid ${ }^{3} \mathrm{He}$ target is given below. The target is described in greater detail in Ref. [11].

Owing to the low intensity of the polarized neutron beam, an extremely thick sample of polarized ${ }^{3} \mathrm{He}$ is desirable. In a measurement of the neutron transmission asymmetry $\varepsilon$, the number of observed neutron counts $N$ necessary to obtain a statistical precision $\Delta \varepsilon / \varepsilon$ is

$$
N=\frac{1}{2}\left[\frac{\Delta \varepsilon}{\varepsilon} \Delta \sigma P_{n} P_{t} \tau\right]^{-2}
$$

The factor of one-half means that $N$ counts are needed in both the up and down spin states. This indicates that, for given values of $P_{n}$ and $\Delta \sigma$, the figure of merit for comparing polarized targets in transmission experiments should be $\tau^{2} P_{t}^{2}$. The figure of merit for the TUNL solid ${ }^{3} \mathrm{He}$ target exceeds current polarized ${ }^{3} \mathrm{He}$ gas targets by nearly two orders of magnitude. Furthermore, the densities of the condensed phases of ${ }^{3} \mathrm{He}$ correspond to nearly $100 \mathrm{MPa}$ of room temperature gas, while targets of polarized ${ }^{3} \mathrm{He}$ gas are limited to $1 \mathrm{MPa}$.

The liquid phase of ${ }^{3} \mathrm{He}$ behaves as a Fermi liquid and can not be polarized to any great extent. On the other hand, solid ${ }^{3} \mathrm{He}$ is a nuclear paramagnet and can be polarized by the static or "brute-force" method; the sample is cooled to a very low $(\sim 10 \mathrm{mK})$ temperature in the presence of an externally-applied magnetic field $(\sim 7 \mathrm{~T})$. The resulting polarization for the body-centered cubic (bcc) phase at temperature $T$ and field $B$ is given by the Brillouin expression

$$
P_{t}=\tanh \left[\frac{1}{k_{B} T}\left(\mu B+\Theta P_{t}+K P_{t}^{3}\right)\right]
$$

where $\mu=-2.13 \mu_{\mathrm{N}}$ is the magnetic moment of the ${ }^{3}$ He nucleus, and $k_{B}$ is Boltzmann's constant. The quantities $\Theta$ and $K$ are corrections to the Curie law of paramagnetism and describe the anti-ferromagnetic exchange of neighboring ${ }^{3} \mathrm{He}$ atoms in the bcc lattice. Therefore, the actual polarization of solid ${ }^{3} \mathrm{He}$ is slightly lower than that calculated assuming simple paramagnetic behavior. Values for these corrections $\left(\Theta / k_{B}=-1.18 \mathrm{mK}\right.$ and $K / k_{B}=$ $-1.96 \mathrm{mK}$ ) were determined by fitting the observed low-temperature properties of bcc solid ${ }^{3} \mathrm{He}$ [12].

For the measurements reported in this paper, a ${ }^{3} \mathrm{He}-{ }^{4} \mathrm{He}$ dilution refrigerator was used to cool the sample to approximately $12 \mathrm{mK}$ in an externally-applied magnetic field of $7 \mathrm{~T}$. The field was provided by a superconducting split-coil magnet operated in persistent-current mode. The magnet was physically rotated to provide fields either parallel (longitudinal) or perpendicular (transverse) to the incident beam direction. The lowest target temperature obtained during these measurements was $11.9 \pm 0.2 \mathrm{mK}$, corresponding to $38.7 \pm 0.6 \%$ polarization.

The sample cell for the target is shown in Fig. 1. The cylindrical container was constructed primarily of beryllium copper $(\mathrm{BeCu})$ with four flat surfaces machined from the cylinder. The flats reduced the amount of material to be cooled and minimized the attenuation of the neutron beam due to $\mathrm{BeCu}$. The wall thickness at the flats was $1.27 \mathrm{~mm}$ 
perpendicular to the beam and $2.54 \mathrm{~mm}$ parallel to the beam. The sample cell was thermally anchored to the dilution refrigerator's mixing chamber by a $45 \mathrm{~cm}$ long OFHC copper cold finger.

The cell was filled with ${ }^{3} \mathrm{He}$ through a $0.75 \mathrm{~mm}$ I.D. stainless steel tube hard-soldered into the top of the cell. Cupro-nickel capillary $(0.1 \mathrm{~mm} \mathrm{I.D.)}$ connected the fill tube to a room temperature gas-handling system. The interior sample space was a rectangular parallelepiped with dimensions $38.1 \times 14.0 \times 21.6 \mathrm{~mm}$ and was filled with 3 micron silver powder packed to $19 \%$ of the density of solid silver. The powder was used to provide good thermal contact between the solid ${ }^{3} \mathrm{He}$ and the $\mathrm{BeCu}$ cell, ensuring a homogeneous temperature throughout the target.

The solid ${ }^{3} \mathrm{He}$ sample was grown by first filling the cell with liquid ${ }^{3} \mathrm{He}$ at approximately $3 \mathrm{~K}$. The liquid was then compressed to a density of $0.125 \mathrm{~g} / \mathrm{cm}^{3}$ by increasing the ${ }^{3} \mathrm{He}$ vapor pressure to $3.6 \mathrm{MPa}$. At this density solid began to form at $1.1 \mathrm{~K}$, and the sample was completely solidified at $0.83 \mathrm{~K}$ [13]. With the silver powder in place, the thickness of the solid ${ }^{3} \mathrm{He}$ sample was $4.34 \pm 0.09 \times 10^{22}$ atoms $/ \mathrm{cm}^{2}$.

The target polarization was extracted from the temperature of the $\mathrm{BeCu}$ sample cell, as measured by two independent thermometric standards: a ${ }^{60} \mathrm{Co}$ Co nuclear orientation thermometer [14] and a ${ }^{3} \mathrm{He}$ melting curve thermometer (MCT) [15]. The nuclear orientation thermometry required an intrinsic germanium detector to observe the $1.17 \mathrm{MeV}$ and $1.33 \mathrm{MeV} \gamma$ rays from ${ }^{60} \mathrm{Co}$. To avoid radiation damage from neutrons however, the detector had to be removed from the experimental hall whenever beam was on target. Therefore the ${ }^{60} \mathrm{Co}$ Co measurements were made immediately before and after each neutron asymmetry measurement. The melting curve thermometer on the other hand, could be used throughout the neutron measurements. The output of the MCT was read directly into the data acquisition computer and the temperature sampled every $100 \mathrm{~ms}$. The average polarization of the target for a particular asymmetry measurement was determined from the average MCT temperature during that time. With no beam on target the ${ }^{60} \mathrm{CoCo}$ and $\mathrm{MCT}$ were found to agree within $\pm 2 \%$, and although the MCT could resolve temperature changes as small as one microkelvin, no significant warming due to neutron or $\gamma$-ray interactions within the target was observed.

\section{B. The Polarized Beam}

\section{Neutron Production and Detection}

Polarized neutrons were produced as secondary beams from either the ${ }^{3} \mathrm{H}(\vec{p}, \vec{n})^{3} \mathrm{He}$ or ${ }^{2} \mathrm{H}(\vec{d}, \vec{n})^{3} \mathrm{He}$ polarization-transfer reactions at $0^{\circ}$. The ${ }^{3} \mathrm{H}(\vec{p}, \vec{n})^{3} \mathrm{He}$ reaction was used to produce neutrons with energy less than $4 \mathrm{MeV}$ because it has a negative Q-value, $-0.764 \mathrm{MeV}$. However, safety considerations limited the maximum amount of tritium that could be used, and the resulting neutron fluxes were low. The ${ }^{2} \mathrm{H}(\vec{d}, \vec{n})^{3} \mathrm{He}$ reaction $(\mathrm{Q}=3.269 \mathrm{MeV})$ was used at higher energies, and the neutron fluxes here were typically 10-20 times greater.

The polarized charged-particle beams were produced by the TUNL atomic beam polarized ion source [16 18] and accelerated by a tandem Van de Graaff. The ion source produced a polarized beam whose spin axis was parallel to its momentum. A Wien filter located between the source and accelerator was used to rotate the polarized beam's spin axis to the 
desired orientation, longitudinal or transverse, at the neutron production target. The position of the beam was feedback-stabilized in both the horizontal and vertical planes by four sets of steering magnets. Computer-controlled steering was used to maintain the beam position at the center of a rotating-wire scanner installed inside the beam pipe approximately $2 \mathrm{~m}$ from the neutron-production target.

The ${ }^{3} \mathrm{H}(\vec{p}, \vec{n})^{3} \mathrm{He}$ neutron production target was a tritiated-titanium foil, backed by a $0.51 \mathrm{~mm}$ thick copper disk. A $0.1 \mathrm{MPa}{ }^{4} \mathrm{He}$ gas cell, with a $2.54 \mu \mathrm{m}$ Havar entrance window, surrounded the tritiated foil to prevent contamination of the beam line. The ${ }^{2} \mathrm{H}(\vec{d}, \vec{n}){ }^{3} \mathrm{He}$ neutron-production target was a deuterium gas cell, $60 \mathrm{~mm}$ long, $19 \mathrm{~mm}$ in diameter and operated at a $\mathrm{D}_{2}$ pressure of $0.4 \mathrm{MPa}$. The Havar window for this cell was $6.35 \mu \mathrm{m}$ thick, and the deuteron beam was stopped by a $0.51 \mathrm{~mm}$ tantalum disk. Both neutron-production targets were air cooled. To eliminate the deflection of the charged particles due to the superconducting magnet, the last $1.2 \mathrm{~m}$ of beam pipe was constructed of soft iron and lined with a high permeability iron-nickel alloy.

The neutron-production targets were located as close as possible to the polarized target. Neutron collimation and detector shielding were accomplished by a combination of copper and polyethylene as shown in Fig. 2. The copper preshield located between the neutron production target and polarized target reduced the number of neutrons striking the superconducting magnet. The polyethylene collimation system located after the polarized target defined a $25.7 \times 9.4 \mathrm{~mm}$ beam spot at the center of the polarized target, corresponding to a solid angle of approximately $0.5 \mathrm{msr}$.

Neutrons that were transmitted through the polarized target were detected by two liquid scintillators located at $0^{\circ}$ and surrounded by a polyethylene shield. The scintillation liquid (BC501) was contained in two cylindrical aluminum containers (127 mm diameter, $127 \mathrm{~mm}$ long), each with an optically transparent endcap coupled to a $127 \mathrm{~mm}$ diameter photomultiplier tube. The cylinders were placed one atop the other, with their axes, as well the photomultiplier tubes, pointing in the vertical direction. Pulse-shape discrimination (PSD) was performed on the phototube anode signals to distinguish neutron events from $\gamma$ rays. The PSD was performed by commercially-manufactured modules [19], with pulseheight thresholds set to discriminate against low-energy neutrons. Valid neutron events were counted in scalers, and stored in the computer at set intervals.

The collimation/detection system was tested in two ways. First, the alignment of the target and collimator was verified by exposing x-ray films to the gammas produced by the charged-particle beam. This showed that the target completely filled the acceptance angle of the collimator. Second, neutron time-of-flight measurements were performed with a pulsed beam at $10 \mathrm{MeV}$. The time-of-flight spectrum showed that only neutrons of the correct energy were being counted. In addition, blocking the exit of the collimator with $30 \mathrm{~cm}$ of tungsten followed by $30 \mathrm{~cm}$ of polyethylene reduced the neutron count rate by a factor of $10^{3}$, indicating that the detectors were adequately shielded from energetic background neutrons.

The neutron transmission asymmetries were observed by reversing the spin of the charged-particle beam every $100 \mathrm{~ms}$. For an accurate measurement it is necessary to know the ratio of the neutron fluxes produced by each spin state of the charged-particle beam. For the ${ }^{3} \mathrm{H}(\vec{p}, \vec{n})^{3} \mathrm{He}$ reaction, the neutron yield is proportional to the proton beam current, and it proved sufficient to count the digitized beam current in each spin state, using the

ratio to normalize the neutron fluxes. Such normalization does not work for the ${ }^{2} \mathrm{H}(\vec{d}, \vec{n}){ }^{3} \mathrm{He}$ 
reaction because here the yield depends on the tensor polarization $P_{z z}$ of the deuteron beam as well as on the beam intensity. The polarized source was operated in a manner such that, ideally, $P_{z z}$ remained constant while the vector polarization was completely reversed. In practice, we determined that the tensor component changed by as much as a few percent when the deuteron spin was flipped. To monitor the flux more reliably, we placed a third liquid scintillator at $0^{\circ}$, between the copper preshield and the ${ }^{3} \overrightarrow{\mathrm{He}}$ target. This monitor detector was used to normalize the number of neutrons in the up and down spin states to the same incident flux. Because of its close proximity to the superconducting magnet, the monitor detector was optically coupled to a $51 \mathrm{~mm}$ diameter phototube by a $1 \mathrm{~m}$ long light pipe. Due to its small dimensions $(25.4 \times 11.1 \times 22.2 \mathrm{~mm}), \gamma$ rays did not deposit much energy in this detector and could be separated from the neutron events by pulse height alone, without the need for pulse-shape discrimination.

Corrections to the incident-flux normalization described above are discussed in Sec. IVB.

\section{Polarization of the neutron beam}

The polarization of the charged-particle beam was measured with a carbon-foil polarimeter. The polarization of the neutron beam was then calculated from the polarization of the charged particles, using known polarization-transfer coefficients. The polarimeter consisted of a thin $\left(5 \mu \mathrm{m} / \mathrm{cm}^{2}\right) 22 \mathrm{~mm}$ diameter carbon foil located at the center of a small scattering chamber. Two silicon surface-barrier detectors detected protons from the ${ }^{12} \mathrm{C}(\vec{p}, p){ }^{12} \mathrm{C}$ reaction, or the ${ }^{12} \mathrm{C}\left(\vec{d}, p_{0}\right){ }^{13} \mathrm{C}$ reaction. The detectors were located at $\pm 40^{\circ}$, and a tantalum collimation system defined a $\pm 3.5^{\circ}$ angular acceptance for each. The carbon foil was mounted to an aluminum plunger inside the polarimeter and was removed from the beam path when not in use.

To determine the polarization of the proton or deuteron beam, a left-right asymmetry was measured between the two silicon detectors. The asymmetry was measured for both the up and down spin states and the difference taken to cancel systematic effects. The average beam polarization was calculated on the basis of this average polarimeter asymmetry, $\varepsilon_{p o l}$ :

$$
\varepsilon_{p o l}=\frac{1}{2}\left[\frac{L^{+}-R^{+}}{L^{+}+R^{+}}-\frac{L^{-}-R^{-}}{L^{-}+R^{-}}\right] .
$$

When polarized protons were used to produce neutrons, the polarimeter measured a left-right asymmetry for the elastic scattering of protons from the carbon foil. Published values 20,21] of the ${ }^{12} \mathrm{C}(\vec{p}, p){ }^{12} \mathrm{C}$ analyzing power $A_{y}$ were then used to calculate the average neutron polarization,

$$
P_{n}=\frac{\varepsilon_{p o l} C_{1} K_{y}^{y^{\prime}}}{A_{y}}
$$

Here $K_{y}^{y^{\prime}}$ (or $K_{z}^{z^{\prime}}$ in the case of a longitudinally polarized beam) is the polarization-transfer coefficient for the ${ }^{3} \mathrm{H}(\vec{p}, \vec{n})^{3} \mathrm{He}$ reaction, and $C_{1}$ is a correction term that describes the depolarization of the neutron beam as it passes through the field of the $7 \mathrm{~T}$ superconducting magnet. The effect is small $\left(C_{1}=0.978\right.$ at $1.94 \mathrm{MeV}$ and $C_{1}=0.984$ at $\left.3.65 \mathrm{MeV}\right)$ because 
the dominant field component is parallel to the neutron spin axis. The values of $C_{1}$ were determined from a detailed calculation of the magnetic field 22].

At the lowest proton energy, $E_{p}=3.0 \mathrm{MeV}$, the ${ }^{12} \mathrm{C}(\vec{p}, p){ }^{12} \mathrm{C}$ analyzing power was too small to be useful as a polarization monitor. Therefore all measurements of the proton polarization were made at $E_{p}=4.7 \mathrm{MeV}$.

When deuterons were used to produce the polarized neutron beam, $P_{n}$ was again determined from the polarimeter asymmetry,

$$
P_{n}=\frac{C_{1} \varepsilon_{p o l}}{A_{e f f}} .
$$

Here $A_{\text {eff }}$ is an "effective" analyzing power that relates the polarimeter asymmetry measured for the ${ }^{12} \mathrm{C}\left(\vec{d}, p_{0}\right){ }^{13} \mathrm{C}$ reaction to the resulting neutron polarization from the ${ }^{2} \mathrm{H}(\vec{d}, \vec{n}){ }^{3} \mathrm{He}$ reaction. This effective analyzing power was measured in a separate experiment with a neutron polarimeter consisting of a ${ }^{4} \mathrm{He}$ scatterer. In this experiment the ${ }^{12} \mathrm{C}\left(\vec{d}, p_{0}\right){ }^{13} \mathrm{C}$ asymmetries were calibrated against known $\vec{n}-{ }^{4} \mathrm{He}$ analyzing powers 23]. The correction factor due to the superconducting magnetic field, $C_{1}$, has been described above, with $C_{1}=$ 0.987 at $4.95 \mathrm{MeV}$ and $C_{1}=0.990$ at $7.46 \mathrm{MeV}$.

During the measurements of $\Delta \sigma_{T}$, the polarimeter asymmetries were measured approximately every $2-3$ hours. Under normal operation of the polarized ion source, we found that the polarimeter asymmetries stayed constant (within experimental uncertainties) during the course of several days. During the $\Delta \sigma_{L}$ measurements, the proton and deuteron polarizations could be measured only after the Wien filter was used to rotate their spins perpendicular to the beam. This involved retuning the beam optics, and so the measurements were performed only twice at each beam energy: immediately before and immediately after the longitudinal neutron-transmission asymmetry was measured.

\section{EXPERIMENTAL PROCEDURE AND DATA ANALYSIS}

\section{A. Measurement of Spin-Spin Asymmetries}

Cooling of the solid ${ }^{3} \mathrm{He}$ target commenced approximately 24 hours before measurements of the neutron-transmission asymmetry. During this time both the ${ }^{60} \mathrm{Co}$ Co nuclear orientation and ${ }^{3} \mathrm{He}$ melting curve thermometers were used to monitor the target temperature. After the target reached a temperature of $15 \mathrm{mK}$, the germanium detector for the ${ }^{60} \mathrm{Co} \underline{\mathrm{Co}}$ thermometer was removed from the experimental hall and the neutron measurements began.

The spin of the neutron beam was reversed every $100 \mathrm{~ms}$ by toggling radio-frequency transition units at the polarized ion source. The spins were flipped according to an eightstep sequence $(+--+-++-)$ to minimize effects which arise from drifts in detector efficiency that are linear or quadratic in time. Typical count rates encountered during these measurements were between $10^{4} \mathrm{~s}^{-1}$ when the ${ }^{2} \mathrm{H}(\vec{d}, \vec{n})^{3} \mathrm{He}$ reaction was used and $400 \mathrm{~s}^{-1}$ with the ${ }^{3} \mathrm{H}(\vec{p}, \vec{n})^{3}$ He reaction.

The data consisted of CAMAC scaler counts of neutron events, digitized charged-particle beam current, digitized polarized target temperature, and events of a $100 \mathrm{kHz}$ dead-time pulser. At the end of each eight-step sequence, a count-down scaler was decremented from 
its preset value of 1024. Data were stored in the computer buffer as spectra of scaler counts versus time, each eight-step sequence comprising one channel of the various spectra. When the count-down scaler reached zero, acquisition was inhibited, and the data were written to the computer disk. All spectra were then cleared, the count-down scaler reset to 1024, and data acquisition recommenced. A "run" therefore consisted of 1024 eight-step sequences and required about fifteen minutes of beam time. At each energy the data were collected for about twelve hours with a polarized target and for an equal amount of time with an unpolarized target.

Two spectra were allocated for each observable of interest, one for neutron-spin up (parallel to target spin), the other for neutron-spin down (antiparallel to target spin). Acquisition into the spin-up spectra was inhibited during the spin-down portions of each eight-step sequence and vice versa. All data acquisition was inhibited $2 \mathrm{~ms}$ prior to and $5 \mathrm{~ms}$ after each spin flip to give the beam polarization time to stabilize. Acquisition was also halted whenever the beam current fell above or below prescribed limits. To ensure that equal time was spent in both the up- and down-spin states, the entire eight-step sequence during which the beam current had fallen outside its limits was rejected in the final data analysis. These occurrences were easily observed in the dead-time pulser spectra. In all, less than $1 \%$ of the data were rejected for this reason.

Transmission asymmetries for the two main neutron detectors were calculated for each eight-step sequence according to

$$
\varepsilon=\frac{\tilde{N}_{+}-\tilde{N}_{-}}{\tilde{N}_{+}+\tilde{N}_{-}} .
$$

Here $\tilde{N}_{ \pm}$is the number of dead time-corrected neutron counts in each spin state normalized to the incident neutron flux,

$$
\tilde{N}_{ \pm}=\frac{N_{ \pm}}{I_{ \pm}}
$$

The normalization factor $I$ is either the proton beam current or the yield in the neutron monitor detector. The transmission asymmetries for all eight-step sequences were combined in a weighted average for both the top and the bottom neutron detectors. These two results were then combined to give the average neutron transmission asymmetry $\bar{\varepsilon}$ and its associated statistical uncertainty. A standard deviation was calculated for each set of data, to compare with the standard deviations expected from Poisson counting statistics.

\section{B. Measurement of Background Asymmetries}

After each measurement of the transmission asymmetry, the solid ${ }^{3}$ He target was melted and warmed to $1 \mathrm{~K}$, and the neutron transmission measurements were repeated. The superconducting magnet continued to operate in persistent-current mode. The polarization of the liquid phase at $1 \mathrm{~K}$ was less than $0.5 \%$, while its density was $8 \%$ less than the density of the

solid. These measurements were performed to determine how much of the observed neutron transmission asymmetry was due to effects other than spin-dependent forces between the polarized neutron beam and polarized target nuclei. 
While these background, or "warm", asymmetries were typically an order of magnitude lower than the spin-spin asymmetries, they were, in general, non-zero and were subtracted from the spin-spin, or "cold" measurements. As discussed below, the asymmetries observed during the warm measurements were due to polarization effects associated with the incident charged-particle beams. As long as the beam polarization remained constant during the warm and cold asymmetry measurements, the background asymmetry was the same in both measurements and so the warm asymmetry could simply be subtracted from the cold. If the polarization differed between the two measurements, a correction based on the two polarizations had to be made. Thus, proper correction for the background asymmetries required some understanding of their origin.

When the ${ }^{3} \mathrm{H}(\vec{p}, \vec{n})^{3} \mathrm{He}$ source reaction was used, the incident proton current provided the normalization factor. However, if imperfect alignment exists between the proton beam and neutron collimation, the vector analyzing power of this reaction will produce a non-zero asymmetry in incident neutron flux that is not eliminated by the beam-current normalization. The vector analyzing power for this reaction vanishes at $0^{\circ}$ and thus the observed background asymmetries (and the subsequent corrections) are small. Since the asymmetry produced by the vector analyzing power is proportional to the polarization of the proton beam $P_{p}$, the background asymmetry observed during the warm measurement is scaled to the same value of $P_{p}$ that existed during the cold measurement. Therefore, $\Delta \sigma_{T}$ is extracted from the difference between the cold and warm asymmetries, with the latter scaled by $P_{p}$,

$$
\Delta \sigma_{T}=\frac{-2}{P_{t} P_{n} \tau}\left[\bar{\varepsilon}_{c}-\frac{P_{p c}}{P_{p w}} \bar{\varepsilon}_{w}\right] .
$$

Here the subscripts $c$ and $w$ refer to the cold and warm measurements, and $P_{n}$ is the value of neutron polarization during the cold asymmetry measurement.

Since parity conservation forbids any longitudinal analyzing powers for the ${ }^{3} \mathrm{H}(\vec{p}, \vec{n})^{3} \mathrm{He}$ reaction, no background correction should be necessary for the low-energy longitudinal measurements. The warm measurement at $3.65 \mathrm{MeV}$ was in fact consistent with zero. Thus $\Delta \sigma_{L}$ (at this energy) was determined from the cold asymmetry measurements alone,

$$
\Delta \sigma_{L}=\frac{-2}{P_{t} P_{n} \tau} \bar{\varepsilon}_{c} .
$$

In the case of the ${ }^{2} \mathrm{H}(\vec{d}, \vec{n})^{3} \mathrm{He}$ reaction we must consider two sources of background asymmetry. In addition to a vector analyzing power (which produces an asymmetry in the neutron yield at non-zero angles), this reaction possesses a tensor analyzing power that affects the neutron yield at $0^{\circ}$. If $I_{0}$ is the $0^{\circ}$ yield from a completely unpolarized deuteron beam, then the yield from a polarized beam, $I\left(0^{\circ}\right)$, will be

$$
I\left(0^{\circ}\right)=I_{0}\left(1-\frac{1}{4} A_{z z} P_{z z}\right)
$$

in the transverse geometry, or

$$
I\left(0^{\circ}\right)=I_{0}\left(1+\frac{1}{2} A_{z z} P_{z z}\right)
$$


in the longitudinal geometry. Here $P_{z z}$ is the longitudinal tensor polarization of the deuteron beam and $A_{z z}$ is the tensor analyzing power for the ${ }^{2} \mathrm{H}(\vec{d}, n)^{3} \mathrm{He}$ reaction. If there is a change in tensor polarization, $\Delta P_{z z}$, when the deuteron spin is flipped at the polarized ion source, then an asymmetry in the $0^{\circ}$ neutron yield will result.

To monitor the $0^{\circ}$ yield, a thin scintillator was placed between the ${ }^{3}$ He target and neutron production target (see Section [IIB). However, the solid angle subtended by the monitor detector was slightly different from that of the main detector. This led to an asymmetry in the monitor-normalized neutron counts caused by either the vector or tensor analyzing power, or both. A vector analyzing power is parity-forbidden for a longitudinally-polarized deuteron beam, and during the transverse measurements we observed little change in the deuteron vector polarization. Therefore the only correction necessary for the ${ }^{2} \mathrm{H}(\vec{d}, \vec{n}){ }^{3} \mathrm{He}$ measurements was one based on the tensor analyzing power. Since both the background asymmetry and the asymmetry observed by the monitor detector were proportional to $\Delta P_{z z}$, it proved convenient to use the monitor asymmetry (which was measured with a high degree of statistical accuracy) to scale the warm to cold asymmetry measurements. Thus, for the ${ }^{2} \mathrm{H}(\vec{d}, \vec{n})^{3} \mathrm{He}$ measurements,

$$
\Delta \sigma_{L, T}=\frac{-2}{P_{t} P_{n} \tau}\left[\bar{\varepsilon}_{c}-\frac{\varepsilon_{m c}}{\varepsilon_{m w}} \bar{\varepsilon}_{w}\right]
$$

where $\varepsilon_{m c}\left(\varepsilon_{m w}\right)$ are the cold (warm) monitor asymmetries.

According to Eqs. 20 and 21, the asymmetry resulting from a given value of $\Delta P_{z z}$ should be twice as large and of the opposite sign in the longitudinal geometry as in the transverse. This explains why the background asymmetries were typically larger (and of the opposite

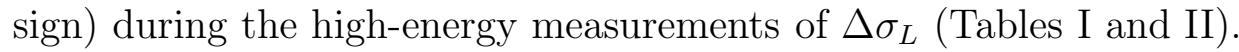

Additional $\Delta \sigma_{T}$ measurements were made with a cold $(\sim 15 \mathrm{mK})$, empty sample container, a cold container filled with liquid ${ }^{3} \mathrm{He}$, and a warm empty container. Such measurements are sensitive to spin-spin effects caused by polarizable materials in the sample container other than ${ }^{3} \mathrm{He}$ (e.g. copper). With the exception of the cold liquid measurements at 1.94 and $3.65 \mathrm{MeV}$, all such background measurements were consistent with the corresponding warm, unpolarized measurements. The asymmetries observed with the cold liquid target were in fact consistent with a ${ }^{3} \mathrm{He}$ polarization of $3 \%$, the expected polarization of ${ }^{3} \mathrm{He}$ in the liquid phase at $12 \mathrm{mK}$ [11,24]. At no time did we observe effects due to polarizable materials other than ${ }^{3} \mathrm{He}$. The only "background" measurement at $4.95 \mathrm{MeV}$ was taken with a cold, empty target. The result here was consistent with zero background asymmetry.

\section{RESULTS}

Transmission asymmetries were measured for the transverse spin geometry at neutron energies of $1.94,3.65,4.95$ and $7.46 \mathrm{MeV}$. The results are given in Table $[$ which includes the corresponding values of the beam and target polarizations. The errors associated with these polarizations are typically $\Delta P_{n} / P_{n}=6 \%$ and $\Delta P_{t} / P_{t}=2 \%$. The uncertainty in $P_{n}$ is dominated by the uncertainty in the ${ }^{12} \mathrm{C}(\vec{p}, p){ }^{12} \mathrm{C}$ and ${ }^{12} \mathrm{C}\left(\vec{d}, p_{0}\right){ }^{13} \mathrm{C}$ polarimeter analyzing powers. Results of the measurements conducted with the target cell filled with cold, liquid ${ }^{3} \mathrm{He}$ (3\% polarization), as well as an empty sample container at both warm $(1 \mathrm{~K})$ and cold

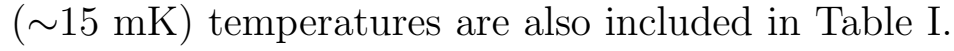


Transmission asymmetries for the longitudinal geometry were measured at neutron energies 3.65, 4.95, and $7.46 \mathrm{MeV}$. A measurement at $1.94 \mathrm{MeV}$ was not attempted because the longitudinal polarization-transfer coefficient for the ${ }^{3} \mathrm{H}(\vec{p}, \vec{n}){ }^{3} \mathrm{He}$ reaction was expected to be too small to produce a useful asymmetry result [25]. The transmission asymmetries at the three higher energies are listed in Table [1] along with their corresponding beam and target polarizations. Here again $\Delta P_{n} / P_{n}=6 \%$ and $\Delta P_{t} / P_{t}=2 \%$. At all three energies the longitudinal asymmetries were considerably smaller than the corresponding transverse asymmetries because the neutron polarizations were lower. Not only did the polarized ion source produce lower charged-particle polarizations during the longitudinal measurements, but the longitudinal transfer coefficients $K_{z}^{z^{\prime}}$ are typically smaller than their transverse counterparts $K_{y}^{y^{\prime}}$.

The values of $\Delta \sigma_{T}$ and $\Delta \sigma_{L}$ extracted from the transmission asymmetries are given in Table [II]. In all but one case, the background asymmetry was taken to be the warm, liquid measurement listed in either Table [I or Table [1]. The cold empty measurement at $4.95 \mathrm{MeV}$ is used for the background correction to $\Delta \sigma_{T}$ at that energy. Both statistical and systematic uncertainties are given. The former reflect the counting statistics associated with a measurement of the transmission asymmetry. The systematic uncertainties are based on uncertainties in beam and target polarizations, as well as target thickness.

\section{COMPARISON TO PHASE-SHIFT PREDICTIONS}

The $\Delta \sigma_{T}$ and $\Delta \sigma_{L}$ results are plotted in Figures 3 and 4 , respectively. The error bars shown in the figures were obtained by adding the systematic and statistical uncertainties in quadrature. For completeness we include the unpolarized neutron total cross section $\sigma_{0}$ in Fig. 5. Experimental results are represented by the ENDF/B-VI polynomial fit (dash-dotted line) [26] to the data of 27 29]. Included in Figures 35 are predictions of $\Delta \sigma_{T}, \Delta \sigma_{L}$, and $\sigma_{0}$ calculated using $n-{ }^{3} \mathrm{He}$ phase shifts obtained from a variety of sources. Briefly, the phase shifts result from two published sets of partial-wave analyses (PWA) of $n-{ }^{3} \mathrm{He}$ scattering data [8.9], a charge-independent $R$-matrix analysis of virtually all $A=4$ scattering and reaction data below excitation energies of $30 \mathrm{MeV}$ [7], and the preliminary results of a microscopic, variational calculation of the ${ }^{4} \mathrm{He}$ continuum [10]. The latter is a multi-channel resonating group model (MCRGM) calculation that uses a gaussian-parameterized version of the Bonn meson-exchange potential [30] as its input. The $\Delta \sigma_{L}$ and $\Delta \sigma_{T}$ calculations have been presented and discussed in greater detail in an earlier paper [5].

Three sets of phase shifts adequately reproduce $\sigma_{0}$, only the MCRGM values are clearly too low. The MCRGM phases also produce values of $\Delta \sigma_{L}$ and $\Delta \sigma_{T}$ that are significantly lower than experiment. All three cases can be attributed to insufficient $P$-wave amplitudes, especially the ${ }^{3} P_{2}$ partial wave. Between neutron energies of 2 and $5 \mathrm{MeV}$, the ${ }^{3} P_{2}$ wave is the dominant partial wave in all four sets of phase shifts, although there is considerable discrepancy as to its strength. The ${ }^{3} P_{2}$ wave of both the MCRGM and the Lisowski PWA are nearly identical to one another, but they are considerably smaller than those of the $R$-matrix or Jany PWA analyses. Consequently these two sets of phase shifts predict the lowest values of both $\Delta \sigma_{L}$ and $\Delta \sigma_{T}$. To correctly reproduce $\sigma_{0}$, the Lisowski PWA compensates for its relatively small ${ }^{3} P_{2}$ wave with unusually large $D$ waves, particularly ${ }^{1} D_{2}$. Since spin-singlet states can only be formed when the beam and target spins are antiparallel to one another, 
Lisowski et al.'s large ${ }^{1} D_{2}$ amplitude further lessen their predictions of $\Delta \sigma_{L}$ and $\Delta \sigma_{T}$.

On the other hand, the Jany PWA possesses the largest ${ }^{3} P_{2}$ wave, ascribing over $60 \%$ of the total (unpolarized) cross section at $2 \mathrm{MeV}$ to this particular wave. Likewise the Jany PWA predicts $\Delta \sigma_{L}$ and $\Delta \sigma_{T}$ values that are slightly higher than experiment.

The $R$-matrix phase shifts reproduce $\Delta \sigma_{T}$ at all four energies. The $R$-matrix prediction of $\Delta \sigma_{L}$ comes closest to the measured values, although it is higher than experiment at $3.65 \mathrm{MeV}$. We see from Fig. 5 that the $R$-matrix also overpredicts the unpolarized total cross section $\sigma_{0}$ by nearly $200 \mathrm{mb}$ at this energy. One possible explanation is the ${ }^{3} P_{1}$ partial wave which, in the $R$-matrix analysis, is much larger at $3.65 \mathrm{MeV}$ than in the other three analyses. While $\Delta \sigma_{T}$ is completely insensitive to this partial wave, $\Delta \sigma_{L}$ is extremely so. If the $R$-matrix $200 \mathrm{mb}$ overprediction of $\sigma_{0}$ is completely attributed to the ${ }^{3} P_{1}$ partial wave, it should likewise overpredict $\Delta \sigma_{L}$ by $400 \mathrm{mb}$ (see Eq. 9). The experimental result of $\Delta \sigma_{L}$ at $3.65 \mathrm{MeV}$ is consistent with this conclusion.

The primary sources of the ${ }^{3} P_{1}$ partial wave are a pair of $1^{-}$resonances at $23.6 \mathrm{MeV}$ $(T=1)$ and $24.2 \mathrm{MeV}(T=0)$. According to the $R$-matrix analysis, both of these excited states are predominately spin-triplet in the nucleon-trinucleon channels. The $\Delta \sigma_{L}$ result at $3.65 \mathrm{MeV}$, in conjunction with the unpolarized neutron total cross section at that energy, may indicate that the resonance parameters associated with one or both of the $1^{-}$levels are in need of slight adjustment.

\section{SUMMARY AND CONCLUSIONS}

We have reported measurements of the polarized neutron-polarized ${ }^{3} \mathrm{He}$ total cross section. A cryogenically-polarized target consisting of nearly $1 / 2$ mole of solid ${ }^{3}$ He has been developed for these measurements. It is the largest sample of polarized ${ }^{3} \mathrm{He}$ yet utilized in a nuclear physics experiment. It is particularly well suited for neutral beams such as neutrons or (real) photons, where the sources of beam-related heating are minimal.

Measurements of the longitudinal and transverse total cross-section differences $\Delta \sigma_{L}$ and $\Delta \sigma_{T}$ were performed for incident neutron energies $2-8 \mathrm{MeV}$. The results are reproduced by phase shifts obtained in a recent $R$-matrix analysis of $A=4$ scattering and reaction data. As such they provide additional support to the ${ }^{4} \mathrm{He}$ level scheme resulting from that analysis. However, the measurement of $\Delta \sigma_{L}$ at $3.65 \mathrm{MeV}$, in conjunction with the unpolarized neutron total cross section at that energy, may indicate that a modification of the $R$-matrix ${ }^{3} P_{1}$ partial wave is necessary.

None of the other three sets of phase shifts considered here are able to reproduce both the present data and previous measurements of the unpolarized neutron total cross section. In all instances we are able to trace the discrepancies to only one or two partial waves. In particular, we find clear evidence that the ${ }^{1} D_{2}$ phase shift reported by Lisowski et al. is too large.

In the future we plan to extend the present measurements to lower energies where the number of partial waves involved in the scattering and reaction processes is limited to two or three. In such circumstances it is possible to uniquely extract all pertinent phase-shift information. 


\section{ACKNOWLEDGEMENTS}

This work was supported in part by the US Department of Energy, Office of High Energy and Nuclear Physics, under contracts DE-FG05-88-ER40441 and DE-FG05-91-ER40619. 


\section{FIGURES}

FIG. 1. The polarized solid ${ }^{3} \mathrm{He}$ target cell. The ${ }^{3} \mathrm{He}$ sample space is indicated by the gray shaded portion. The interior dimensions of the target are given on the right.

FIG. 2. Diagram of the experimental apparatus showing the neutron-production target, polarized target, collimation, and neutron detectors.

FIG. 3. Measured values of $\Delta \sigma_{T}$ (triangles). Error bars indicate systematic and statistical

uncertainties added in quadrature. Also shown are phase-shift predictions of $\Delta \sigma_{T}: R$ matrix (solid line), MCRGM (dashed line), Jany PWA (diamonds) and Lisowski PWA (circles).

FIG. 4. Measured values of $\Delta \sigma_{L}$. Symbols same as Fig. 3.

FIG. 5. Phase-shift calculations of the unpolarized neutron total cross section $\sigma_{0}$. Symbols

same as Fig. 3. The ENDF/B-VI polynomial fit [26] to the experimental data of [27 29] is also given (dash-dotted line). 


\section{TABLES}

TABLE I. Results of transverse neutron-transmission asymmetries. Here $P_{t}$ and $P_{n}$ are the ${ }^{3} \mathrm{He}$ and neutron polarizations, respectively. The uncertainty quoted for each transmission asymmetry $\bar{\varepsilon}$ reflects counting statistics, while $\sigma_{\bar{\varepsilon}}$ is the reduced standard deviation for all the eight-step sequences corresponding to a measurement of $\bar{\varepsilon}$. In the case of the ${ }^{2} \mathrm{H}(\vec{d}, \vec{n})^{3} \mathrm{He}$ measurements, $\varepsilon_{m}$ is the asymmetry observed in the neutron monitor detector.

\begin{tabular}{clccrrr}
\hline \hline$E_{n}(\mathrm{MeV})$ & Target Cell & $P_{t}$ & $P_{n}$ & $\bar{\varepsilon}\left(10^{-4}\right)$ & $\sigma_{\bar{\varepsilon}}\left(10^{-4}\right)$ & $\varepsilon_{m}\left(10^{-4}\right)$ \\
\hline \multirow{2}{*}{1.94} & cold solid & 0.365 & 0.482 & $-42.94 \pm 1.72$ & 1.86 & - \\
& cold liquid & 0.029 & 0.482 & $-5.12 \pm 1.90$ & 2.62 & - \\
& warm liquid & 0.000 & 0.482 & $3.15 \pm 2.15$ & 2.34 & - \\
\multirow{3}{*}{3.65} & cold solid & 0.351 & 0.530 & $-45.52 \pm 2.16$ & 2.50 & - \\
& cold liquid & 0.029 & 0.500 & $-5.90 \pm 2.27$ & 2.58 & - \\
& warm liquid & 0.000 & 0.492 & $0.66 \pm 2.09$ & 2.40 & $68.33 \pm 0.54$ \\
\multirow{3}{*}{7.95} & cold solid & 0.307 & 0.521 & $-30.22 \pm 1.05$ & 1.04 & $58.49 \pm 0.53$ \\
& cold empty & 0.000 & 0.521 & $-0.98 \pm 0.98$ & 0.97 & $51.71 \pm 0.40$ \\
& cold solid & 0.345 & 0.632 & $-23.57 \pm 0.64$ & 0.65 & $54.66 \pm 0.35$ \\
& cold empty & 0.000 & 0.639 & $-2.51 \pm 0.57$ & 0.57 & $66.81 \pm 0.39$ \\
& warm empty & 0.000 & 0.634 & $-3.76 \pm 0.64$ & 0.64 & $54.73 \pm 0.33$ \\
\hline \hline
\end{tabular}

TABLE II. Results of longitudinal neutron-transmission asymmetries.

\begin{tabular}{clccrrr}
\hline \hline$E_{n}(\mathrm{MeV})$ & Target Cell & $P_{t}$ & $P_{n}$ & $\bar{\varepsilon}\left(10^{-4}\right)$ & $\sigma_{\bar{\varepsilon}}\left(10^{-4}\right)$ & $\varepsilon_{m}\left(10^{-4}\right)$ \\
\hline \multirow{2}{*}{3.65} & cold solid & 0.351 & 0.263 & $-8.65 \pm 1.89$ & 1.91 & - \\
& warm liquid & 0.000 & 0.265 & $1.81 \pm 1.92$ & 1.95 & - \\
\multirow{2}{*}{4.95} & cold solid & 0.352 & 0.334 & $-18.81 \pm 1.17$ & 1.19 & $263.4 \pm 0.87$ \\
& warm liquid & 0.000 & 0.372 & $2.16 \pm 1.19$ & 1.22 & $250.2 \pm 0.75$ \\
\multirow{2}{*}{7.46} & cold solid & 0.343 & 0.404 & $-6.34 \pm 0.60$ & 0.60 & $127.0 \pm 0.48$ \\
& warm liquid & 0.000 & 0.210 & $4.37 \pm 0.62$ & 0.63 & $134.7 \pm 0.47$ \\
\hline \hline
\end{tabular}

TABLE III. Results of the $\Delta \sigma_{L}$ and $\Delta \sigma_{T}$ measurements. The first uncertainty is systematic, the second statistical.

\begin{tabular}{ccc}
\hline \hline$E_{n}(\mathrm{MeV})$ & $\Delta \sigma_{L}(\mathrm{~b})$ & $\Delta \sigma_{T}(\mathrm{~b})$ \\
\hline 1.94 & - & $1.207 \pm 0.092 \pm 0.078$ \\
3.65 & $0.432 \pm 0.044 \pm 0.095$ & $1.145 \pm 0.089 \pm 0.089$ \\
4.95 & $0.806 \pm 0.068 \pm 0.067$ & $0.838 \pm 0.073 \pm 0.044$ \\
7.46 & $0.348 \pm 0.041 \pm 0.028$ & $0.431 \pm 0.035 \pm 0.018$ \\
\hline \hline
\end{tabular}




\section{REFERENCES}

[1] W. Glöckle, H. Witala, H. Kamada, D. Hüber, and J. Golak, in Proceedings of the XIV International Conference on Few Body Problems in Physics, Williamsburg, VA., edited by F. Gross (American Institute of Physics, New York, 1995), pp. 45-67.

[2] D. R. Tilley, H. R. Weller, and G. M. Hale, Nucl. Phys. A541, 1 (1992). This compilation article contains a complete reference guide to experimental and theoretical work on $A=4$ nuclei since 1973 .

[3] L. Passell and R. I. Schermer, Phys. Rev. 150, 146 (1966).

[4] M. T. Alley and L. D. Knutsen, Phys. Rev. C 48, 1890 (1993).

[5] C. D. Keith, C. R. Gould, D. G. Haase, G. M. Hale, H. M. Hofmann, H. Postma, N. R. Roberson, and W. Tornow, Phys. Rev. C 50, 237 (1994).

[6] C. D. Keith, C. R. Gould, D. G. Haase, P. R. Huffman, N. R. Roberson, M. L. Seely, W. Tornow, and W. S. Wilburn, in Proceedings of the XIV International Conference on Few Body Problems in Physics, Williamsburg, VA., edited by F. Gross (American Institute of Physics, New York, 1995), pp. 439-442.

[7] G. M. Hale, D. C. Dodder, and K. Witte, $A=4$ R-matrix analysis of 9/88 (unpublished).

[8] P. Jany, W. Heeringa, H. O. Klages, B. Zeitnitz, and R. Garrett, Nucl. Phys. A483, 269 (1988).

[9] P. W. Lisowski, R. L. Walter, C. E. Busch, and T. B. Clegg, Nucl. Phys. A242, 298 (1975).

[10] H. M. Hofmann, unpublished.

[11] C. D. Keith, C. D. Gould, D. G. Haase, P. R. Huffman, N. R. Roberson, M. L. Seely, W. Tornow, and W. S. Wilburn, Nucl. Inst. and Meth. A 357, 34 (1995).

[12] H. L. Stipdonk and J. H. Hetherington, Phys. Rev. B 31, 4684 (1985).

[13] E. R. Grilly, J. Low. Temp. Phys. 4, 615 (1971).

[14] H. Marshak, J. of Res. of the NBS 88, 175 (1983).

[15] C. D. Keith, C. D. Gould, D. G. Haase, N. R. Roberson, W. Tornow, and W. S. Wilburn, Hyperfine Interactions 75, 525 (1992).

[16] T. B. Clegg, W. M. Hooke, E. R. Crosson, A. W. Lovette, H. L. Middleton, H. G. Pfutzner, and K. A. Sweeton, Nucl. Inst. and Meth. A 357, 212 (1995).

[17] T. B. Clegg et al., Nucl. Inst. and Meth. A 357, 200 (1995).

[18] D. C. Dinge, T. B. Clegg, E. R. Crosson, and H. W. Lewis, Nucl. Inst. and Meth. A 357, 195 (1995).

[19] Link Analytical Limited, Bucks, England.

[20] S. J. Moss and W. Haeberli, Nucl. Phys. 72, 417 (1965).

[21] G. E. Terrell, M. F. Jahns, M. R. Kostoff, and E. M. Bernstein, Phys. Rev. 173, 931 (1968).

[22] W. S. Wilburn, C. R. Gould, D. G. Haase, P. R. Huffman, C. D. Keith, N. R. Roberson, and W. Tornow, Phys. Rev. C 52, 2351 (1995).

[23] W. Tornow, Z. Physik 266, 357 (1974).

[24] H. Ramm, P. Pedroni, J. R. Thompson, and H. Meyer, J. Low. Temp. Phys. 2, 539 (1970).

[25] J. J. Jarmer, J. C. Martin, G. G. Ohlsen, G. C. Salzman, and J. E. Simmons, Phys. Lett. 48B, 215 (1974). 
[26] G. Hale, D. Dodder, and P. Young, ENDF/B-VI Data File for ${ }^{3} H e$, National Nuclear Data Center, Brookhaven National Laboratory, Upton, New York, 1991.

[27] Los Alamos Physics and Cryogenics Group, Nucl. Phys. 12, 291 (1959).

[28] C. A. Goulding, P. Stoler, and J. D. Seagrave, Nucl. Phys. A215, 253 (1973).

[29] B. Haesner, W. Heeringa, H. O. Klaages, F. Käppeler, G. Schmalz, P. Schwarz, J. Wilczynski, B. Zeitnitz, and H. Dobiasch, Phys. Rev. C 28, 995 (1983).

[30] H. Kellermann, H. M. Hofmann, and C. Elster, Few-Body Sys. 7, 31 (1989). 


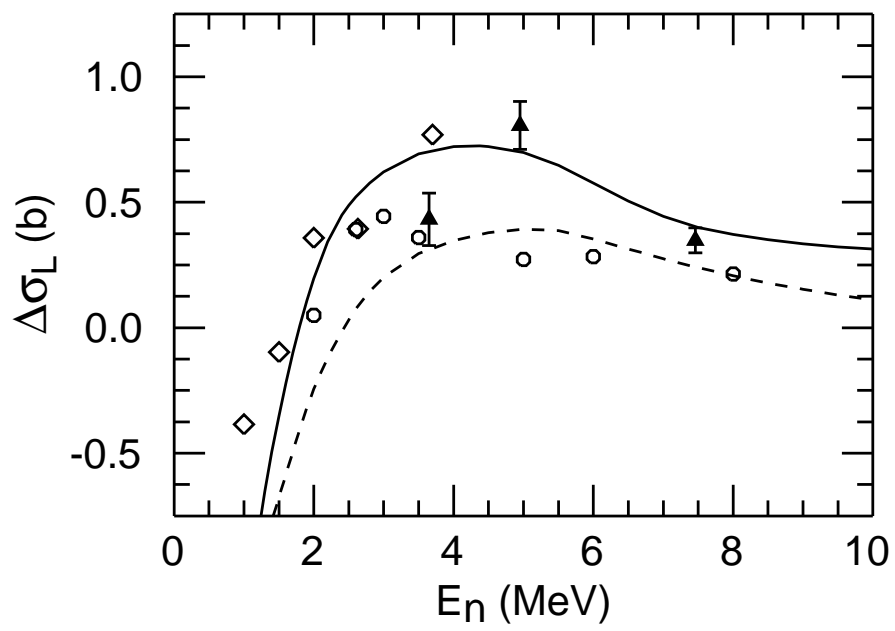




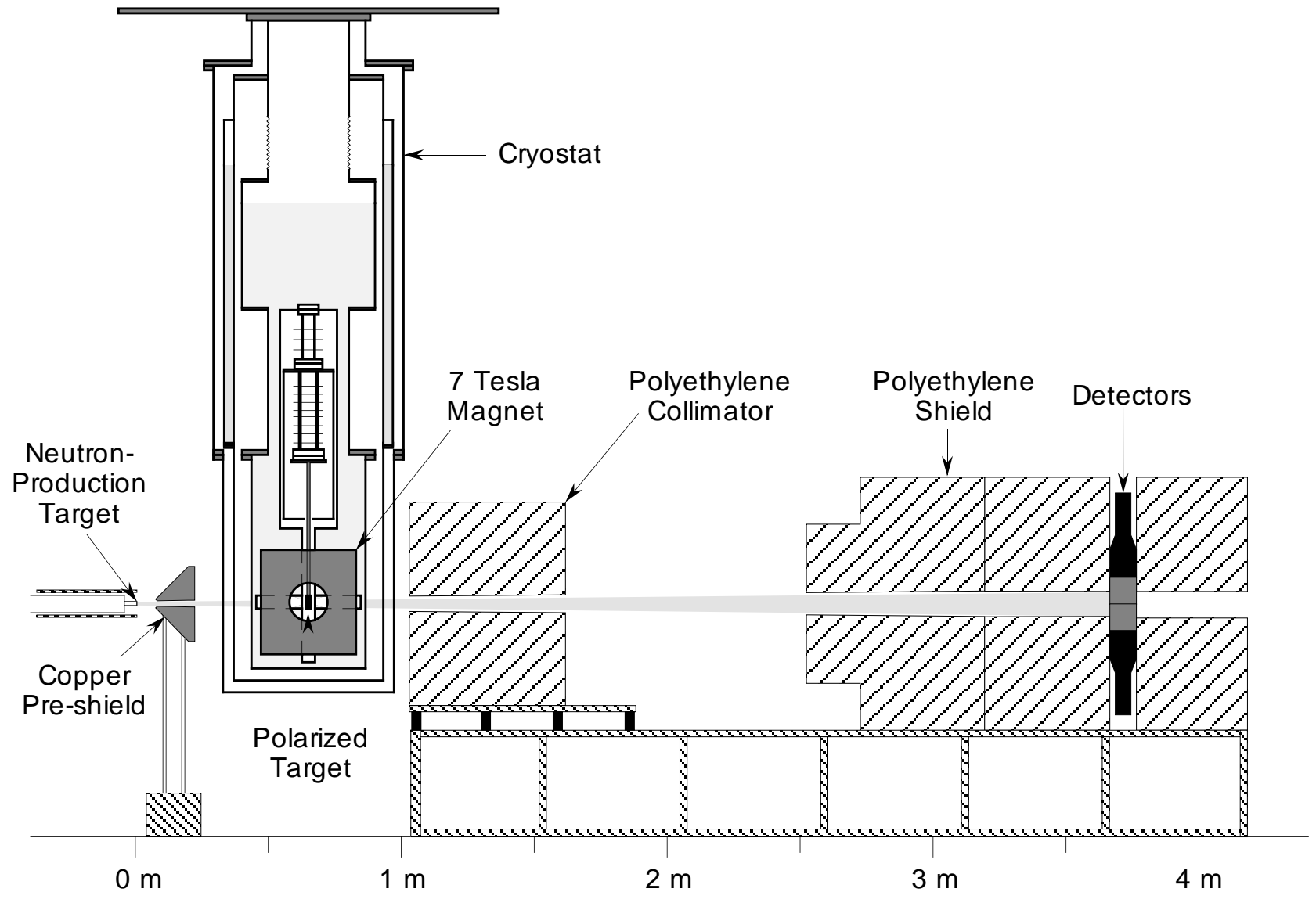




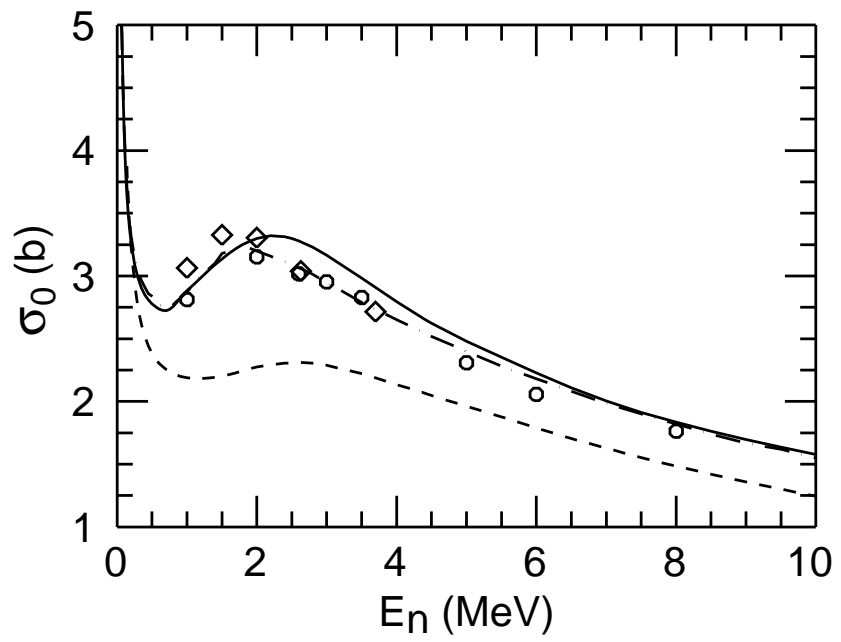




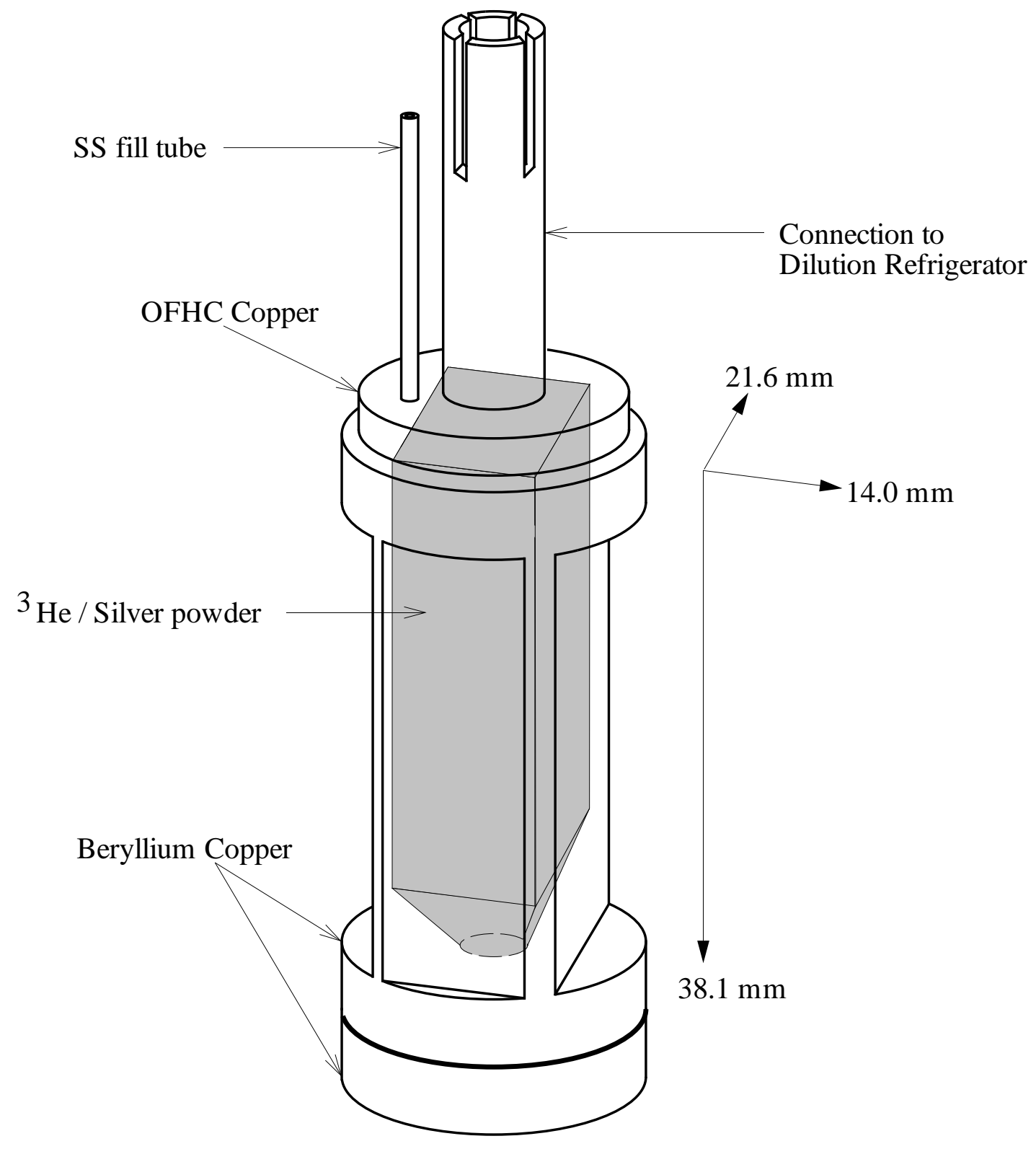




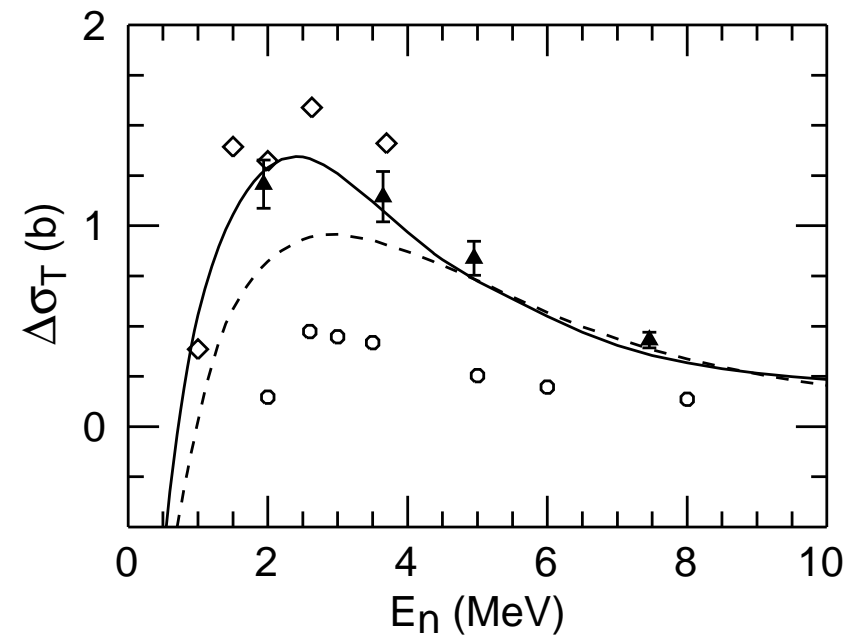

